\title{
COMPARATIVE STUDY OF POWDER CELLULOSES AND CELLULOSE HYDROGELS BY WAXS METHOD. IMPACT OF MEASUREMENT TECHNIQUE AND COMPUTATION ON VARIABILITY OF RESULTS
}

\author{
SHAHRIAR KARIM SAUROV, ${ }^{*}$ ALEKSANDRA MIKHAILIDI, ${ }^{* *}$ KIRSI SVEDSTRÖM* $^{*}$ and \\ NINA KOTELNIKOVA*** \\ "Department of Physics, University of Helsinki, Helsinki FI-00014, Finland \\ ${ }^{* *}$ St. Petersburg State University of Industrial Technologies and Design, St. Petersburg 191186, Russia \\ ${ }^{* * *}$ Institute of Macromolecular Compounds, Russian Academy of Sciences, \\ St. Petersburg 199004, Russia \\ $₫$ Corresponding author: A. Mikhailidi, amikhailidi@yahoo.com
}

Dedicated to the anniversary of the Department of Pulp and Paper founded by Acad. Prof. Cr. I. Simionescu at the Polytechnic Institute of Iasi, Romania

\begin{abstract}
A comparative study of crystallinity and size of cellulose crystallites of powder celluloses from hardwood pulp, flax fibers and cellulose hydrogels was performed by the wide-angle X-ray scattering method (WAXS). Cellulose hydrogels were prepared by regeneration from solutions of powder celluloses in DMAc/LiCl. The variation in the structural characteristics of the swollen hydrogels and the freeze-dried hydrogels, alongside the powder celluloses, was studied. The swollen hydrogels contained over 97 mass\% water, and thus their WAXS patterns resembled those of water. The WAXS patterns for both powder celluloses indicated cellulose I structure, whereas the freeze-dried hydrogels revealed cellulose II polymorph. The impact of the calculation mode on the obtained crystallinity values was investigated using three analysis methods. The Segal method could be used only for cellulose I samples, whereas amorphous fitting and amorphous subtraction methods could be applied for both samples, cellulose I and II. The Segal method gave the highest crystallinity values, while the amorphous fitting and the amorphous subtraction generated similar values. The advantages of using the amorphous fitting method and the effect of drying on the structure of the hydrogels were discussed.
\end{abstract}

Keywords: cellulose, hydrogels, crystallinity, wide-angle X-ray scattering, amorphous fitting method

\section{INTRODUCTION}

Cellulose is made up of long chains of repeating $\beta$-(1-4)-linked-D-glucopyranose units, shaping a glycan chain, which can be chemically modified to get various types of derivatives and composites that have valuable properties. ${ }^{1}$ This polysaccharide offers a wide range of eco-friendly applications in different areas of our life, such as printing, cosmetics, detergents, enhancing oil recovery, textiles, medicine and pharmacy. ${ }^{2,3}$ Cellulose is known to belong to a class of capillary-porous systems containing a highly branched inner and outer surface of macro- and micropores, as well as dead-end pore voids. These properties promote the adsorption of solvents or water on the surface and/or their penetraton into the bulk. However, because of the strong intraand intermolecular hydrogen bonds in native cellulose chains, these phenomena are restricted and cellulose has limited absorption capacity, also, cellulose is not soluble in most common solvents, including water. ${ }^{4}$ This specificity influences the main properties of cellulose. To dissolve cellulose, different kinds of solvents have been tested, but many of them have failed from a commercial viewpoint because of byproduct formation, toxicity to the environment and instability of the process. The solvent DMAc/LiCl, having chloride as hydrogen bond acceptor, is one of the effective solutions for organic synthesis based on the cellulose. When 
natural cellulose I dissolves in $\mathrm{DMAc} / \mathrm{LiCl}$ solution, $\mathrm{Cl}^{-}$substitutes the $\mathrm{OH} \cdots \mathrm{H}$ bond with the $\mathrm{OH} \cdots \mathrm{Cl}$ bond and $\mathrm{Li}^{+}$forms a complex structure $\mathrm{Li}^{+}(\mathrm{DMAc})_{\mathrm{x}}$. The dissolution proceeds due to a rupture of inter- and intra-molecular $\mathrm{H}$-bonds in the cellulose chains. Then, regenerated from the solution, cellulose undergoes structural modification and converts from the less stable parallel arrangement of cellulose I to a more stable anti-parallel arrangement of cellulose II. ${ }^{4-6}$

In the last decades, novel cellulose-based materials, such as hydrogels, have attracted much attention and were extensively investigated because these biodegradable systems have numerous advantages. Hydrogels are usually prepared from solutions of different cellulose samples in various solvents, followed by subsequent regeneration from the solutions. They consist in one or more three-dimensional highly hydrated polymeric networks, formed from macromolecular chains interconnected by chemical (covalent) bonds or physical interactions. The hydrogels can retain large amounts of water compared to the pristine cellulose. ${ }^{7}$ Out of the studies dedicated to hydrogels, the majority have been made on those derived from bacterial cellulose, due to the peculiarities of the synthesis and shape. ${ }^{8}$ In recent years, plant-derived cellulose has come to be also widely applied to prepare hydrogels., ${ }^{7-11}$ The unique properties of hydrogels, namely, their high water-holding and absorption capacity, as well as sufficiently high stiffness, were actively studied by various physical methods. ${ }^{12,13}$ Some major aspects concerning hydrogels have been considered in numerous publications, including a number of reviews (see for instance ${ }^{14}$ ). The first aspect concerned the mechanism of preparation of hydrogels via regeneration from the solutions of pristine natural celluloses. Many attempts have been also made to characterize the structure of the hydrogels with various methods, including WAXS.

One of the most important parameters to characterize the crystalline structure of cellulose samples and changes of the structure is the crystallinity value. While the detailed supermolecular structure of cellulose is still a matter of debate, different methods have been applied to define this value. A widely used method for measuring the crystallinity of cellulose samples is the X-ray diffraction method based on scattering X-rays. One of the X-ray scattering techniques is wide-angle $\mathrm{X}$-ray scattering (WAXS). The WAXS technique has been used in our study due to its sensitivity towards small structural changes and robustness in obtaining data. Using this technique, the averaged data could be obtained from a relatively large sample. To calculate the crystallinity, it is necessary to take into account a number of important factors. In this study, we have focused on some points that should be taken into consideration. Different analysis methods have been developed over the years for measuring crystallinity, such as the peak height method, commonly known as the Segal method, the Gaussian peaks method and the amorphous subtraction method, beside others (see e.g. ${ }^{15}$ ). It is important to select a proper method as the crystallinity of a single sample may vary a lot (e.g. even between 37-93\%), depending on the calculating method, sample morphology and data collection. ${ }^{16}$ Here, we display shortly the most popular methods and we focused on the most important points considering the different cellulose samples of this study. Crystallinity determination with three analysis methods are shown in Figure 1.

\section{Peak height method}

One of the most frequently used methods for calculating crystallinity is the peak height method because the calculation is simple. ${ }^{15,17}$ This method was developed by L. Segal and co-workers, and considered only one reflection of cellulose I lattice (200), which appears between $2 \theta=22^{\circ}-23^{\circ}$, and ignores all other cellulose reflections, including the 110 and $1-10$ peaks. In this method, crystallinity is calculated by comparing the intensity of the 200 peak to the intensity at the mimimum between the 200 and $1-10$ peaks(Fig 1a). ${ }^{18}$

The crystallinity is obtained as:

$C=\left(I_{200}-I_{\min }\right) / I_{200}$

where $\mathrm{I}_{200}$ is the intensity of the 200 peak and $\mathrm{I}_{\min }$ is the minimum intensity.

There is also an extended Segal method for cellulose II samples, in which instead of the minimum around $18^{\circ}$, a minimum around $16^{\circ}$ is considered, and instead of the intensity of the 200 peak of cellulose I, the intensity of the 020 peak of cellulose II (around $21.7^{\circ}$ ) is considered. ${ }^{19,20}$

This method has the tendency to provide higher crystallinity values and neglects the effects of the variation in the crystalline widths on the crystallinity. ${ }^{15,20}$ However, the method is suitable 
to estimate the crystalline state of different materials inside a sample series, i.e. compares the crystallinity between similar kinds of samples rather than giving the actual crystallinity values. ${ }^{15}$

\section{Amorphous subtraction method}

Another widely used method is the RulandVonk method, ${ }^{21}$ also known as amorphous subtraction method. In this method, the scattering pattern of an amorphous sample (or a corresponding model curve) is fitted under the intensity curve of the studied sample and subtracted from the total intensity (Fig 1b). The crystallinity of the samples is calculated by using the amorphous contribution and the intensity of the entire sample by the following equation drafted by Ruland: ${ }^{21}$

$\mathrm{C}=1-\left(\int \mathrm{I}_{\text {amor }} / \int \mathrm{I}_{\text {sample }}\right)=\mathrm{I}_{c r} / \mathrm{I}_{\text {sample }}$

where $\mathrm{I}_{\text {amor }}$ is the amorphous intensity and $\mathrm{I}_{\text {sample }}$ is the total intensity of the sample. So, this method is based on the fact that the X-ray scattering intensities are quantitative values representing the volume fractions of the scattering components (different crystalline or amorphous allomorphs of the sample).

\section{Amorphous fitting method}

The amorphous fitting method is similar to the amorphous subtraction method, however, both the crystalline and the amorphous components are modelled in this method (Fig. 1c). Due to this, for the improvement of fitting, the fitted amorphous contribution could exceed sample intensities at some scattering angles. This can happen due to differences in the actual shape of the amorphous contribution and the selected amorphous standard. Nuclear magnetic resonance spectroscopy (NMR) is also a powerful tool of measuring the crystallinity of cellulose. ${ }^{22}$ A study on cellulose has shown that the amorphous fitting method corresponds best with the NMR results, compared to other methods. Another positive aspect of using this method is that it is the least vulnerable to crystallite size, compared to other common crystallinity calculating methods. ${ }^{15}$

Finding a suitable amorphous model for the standard amorphous component is the most crucial requirement of this fitting method because it will have a direct impact on the crystallinity values. A substance that is chemically equivalent to the studied sample shoud be selected as the amorphous material. One of the most widely used substances for the standard amorphous component is ball milled cellulose. In our study, we used lignin as the standard amorphous material. ${ }^{23}$ The reasons for choosing lignin as the standard amorphous material is that it has been experimentally verified as a suitable amorphous model in numerous studies on various plant, wood and cellulose based samples. ${ }^{15,23,24}$

The present paper aimed to address two main issues. We focused on the comparative exploration of the sample in frames of sets from the powder cellulose samples to the samples regenerated from the dissolved state in DMAc/LiCl solutions, in the form of hydrogels, with WAXS. Three different calculating techniques, namely, amorphous fitting, amorphous subtraction and peak height methods, were used for calculating the crystallinity of the samples. A comparison of the results computed in various calculation modes was the second objective of the study. Behind crystallinity calculation of cellulosic samples, there are many important factors: variation of crystallinity values depending on the plant sample origin, data collection and data processing, the structural transition from cellulose powder to cellulose hydrogels and the drying of the swollen hydrogels on the cellulose structure. Thus, the aim was to study all these factors comparatively.

\section{EXPERIMENTAL}

\section{Materials and sample preparation}

The powder samples were isolated from two different pristine samples: short flax fibers (FP) and bleached sulfite hardwood pulp (HP). The hardwood pulp was purchased from Syktyvkar Timber Plant ("Mondi", Syktyvkar, Russia) and the flax fibers were linseed waste.

The preparation of the powder celluloses, including the hydrolysis of the pristine samples, the dissolution of the powders in the solutions of $\mathrm{DMAc} / \mathrm{LiCl}$ and the regeneration from the solutions, was performed as described elsewhere. ${ }^{25}$ Shortly, the pulp and the flax fibers were hydrolyzed with a $10 \mathrm{wt} \%$ aqueous solution of nitric acid to prepare the powder cellulose samples. The dissolution of cellulosics in DMAc/LiCl strongly required a pretreatment of the samples, which was performed by solvent exchange, by infusing and rinsing the powder celluloses with deionized water and then with DMAc. The concentration of both samples in the solutions was kept at $3.0 \mathrm{wt} \%$. The dissolving procedure included heating of the pretreated samples in the solution of DMAc to $165^{\circ} \mathrm{C}$. $\mathrm{LiCl}$ was added to the suspension to obtain a solution with the concentration of $\mathrm{LiCl}$ of $8.0 \mathrm{wt} \%$. The mixture was kept under stirring, yielding clear (for powder pulp cellulose) or semi-clear (for powder flax cellulose) solutions, which were cooled to room temperature and filtered through a 
glass filter. To monitor the gelation of the regenerated samples, the solutions were poured into dishes and left under ambient conditions for 5-7 days. The gels were formed by absorbing the solvent and finally, the swollen samples did not change anymore. The prepared gels were rinsed with water, herewith the solvent was replaced with the water. Thus, the formation of the hydrogels was performed without any anticoagulants, due to a spontaneous self-assembly and aggregation of cellulose chains. This is the main distinction of our method from those described earlier. $^{2,6}$

The shape and properties of the regenerated hydrogels strongly depended on a number of parameters, such as the concentration of the solutions, the regenerating method during the absorption of the solvent and/or subsequent aggregation, time for gel formation and drying procedure. The main physicochemical properties of the obtained hydrogels were studied and described in our earlier publication. ${ }^{25}$ To characterize the sustainability of the hydrogels, we investigated their long-term stability while varying time and temperature of storing in water and ethyl alcohol, separately or combined in various proportions, by controlling their transparency depending on the composition of the aqueous solutions. We also monitored the absorbing capacity and swelling values of the hydrogels during storage in the above solutions.
The hydrogels were freeze-dried as freeze-drying maintains the properties of organic compounds unchanged and thus provides the possibility to study their structure, without any interference.

All the chemicals, including anhydrous $\mathrm{LiCl}$, purum p.a., purchased from Fluka, and N,Ndimethylacetamide, purchased from Sigma-Aldrich, were of analytical grade.

\section{Sample preparation for measurements}

Every set of samples contained three samples: the powder sample, the swollen hydrogel regenerated from the solution of the powder and the freeze-dried hydrogel. An example of one set of the samples is imaged in Figure 2 (a-c). The powder samples were ground to fine particles under pressure, then pressed into a pellet and put into aluminum rings for measurement. Uniform thickness of the sample was ensured. The hydrogels were measured in the swollen state and were also dried using the freeze-dry technique. The swollen and freeze-dried hydrogels were measured as they were, because any changes in the morphology of the hydrogels could lead to alteration of their morphological or crystalline structure and crystal width. All measurements were duplicated.
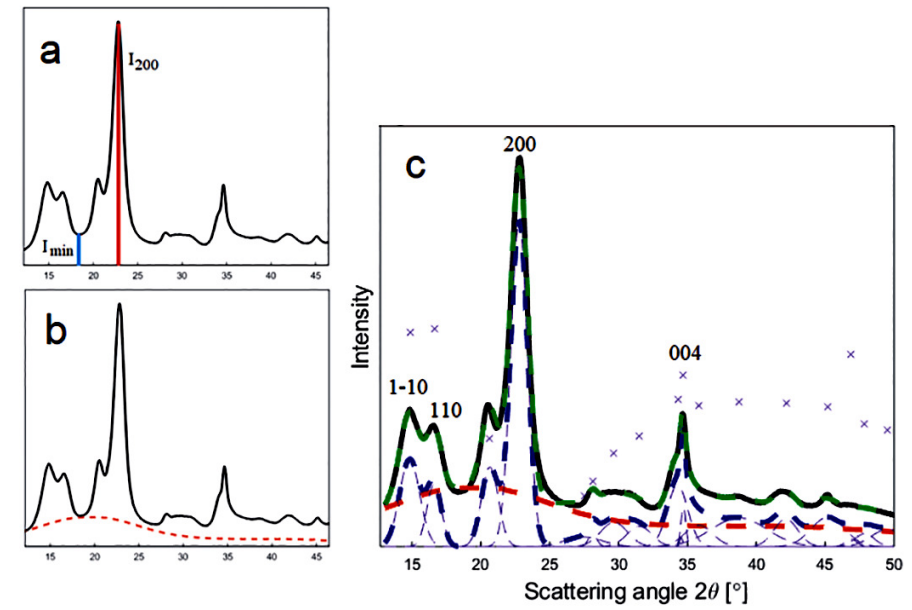

Figure 1: Crystallinity determination with three analysis methods, (a) peak height method, (b) amorphous subtraction method and (c) amorphous fitting method
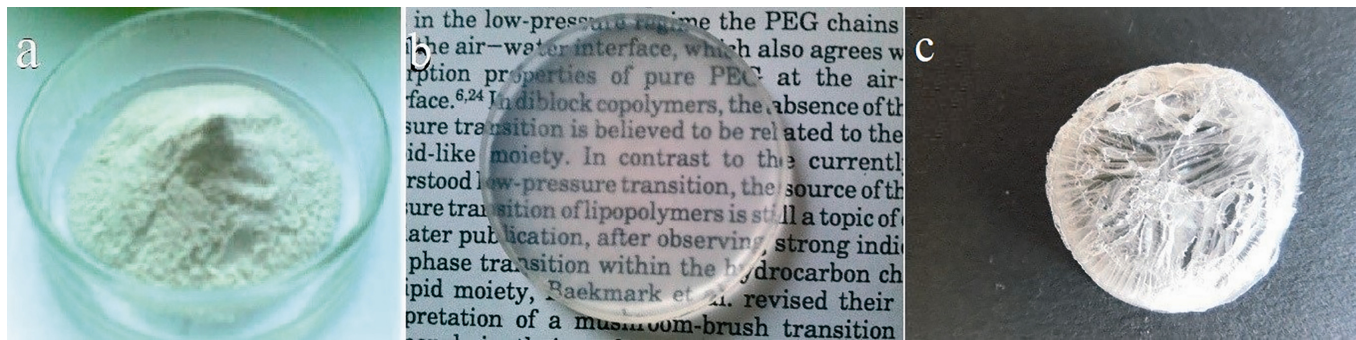
Figure 2: Digital photos of the samples prepared from hardwood pulp fibres: (a) powder cellulose, (b) hydrogel and (c) freeze-dried hydrogel

\section{Wide-angle $X$-ray scattering}

WAXS measurements were performed to compute the crystallinity and average crystallite width of the cellulose samples. The WAXS setup at the University of Helsinki consists of a sealed X-ray copper tube, which generates monochromatic $\mathrm{CuK} \alpha$ radiation with the wavelength of $1.541 \AA(\lambda)$, and a two-dimensional MAR345 image plate (Marresearch $\mathrm{GmbH}$, Norderstedt, Germany) for detection, i.e. the measurements were conducted using the perpendicular transmission geometry. The distance between the sample and the image plate was $120 \mathrm{~mm}$. By using slits, the beam size was adapted to be $0.5 \times 0.55 \mathrm{~mm}^{2}$. The length of the scattering vector ( $q$-range) was calibrated using a calibration sample lanthanum hexaboride and was found to be 0.3-3.4 $\AA^{-1}$, corresponding to the scattering angle range of $4.2-50^{\circ}$. The relation between the $q$ values and the scattering angle $2 \theta$ is:

$q=4 \pi \sin (\theta) / \lambda$

Each sample was measured for 15-30 minutes. The obtained diffraction patterns of the sample need corrections as numerous factors have an influence on the measured intensity: these corrections include dark current, air scattering, polarization, as well as sample absorption corrections. The absorption correction was based on the transmission values measured by a semitransparent copper beamstop, and the background due to air scattering was subtracted after the absorption correction. All these corrections were carried out before calculating the crystallinity values.

\section{Crystallinity calculation}

The crystallinity of the cellulose samples was computed in three ways, using the peak height method, amorphous subtraction and amorphous fitting analysis methods, as explained in Introduction. For the amorphous subtraction method, the lowest used value of the scattering angle was $10.5^{\circ}$ and the highest was $49.5^{\circ}$. For the amorphous fitting method, the crystalline pattern was modelled as a sum of Gaussian peaks in the positions of cellulose I reflections in the used range of scattering angle from $13.0^{\circ}$ to $50.0^{\circ}$ (see Fig. 3). For freeze-dried hydrogel samples, the main reflections of cellulose II, i.e. reflections 1-10, 110 and 020 , were also included in the model. ${ }^{26}$

\section{Crystallite size calculation}

In a crystalline material, crystals have a finite extension and the diffracted beam is spread over a region instead of being confined to a point. The size and shape of that spread region is related to the crystal size of that material. The crystallite size influences the width of the diffraction peak, which has been established by Scherrer by the following equation: ${ }^{27}$
$\mathrm{L}=K \lambda / B \cos \theta$

where $K$ represents a constant that is taken to be $0.9, \lambda$ is the wavelength (1.541 $\AA$ ), $\theta$ is Bragg's angle and $B$ is the FWHM (full width at half maxima) of the peak. For this calculation, the cellulose 200 reflection was considered for cellulose I samples, and in the case of cellulose II, the 110 reflection was used.

For calculating the average crystal size, the instrumental broadening effect has to be considered. This effect arises for various reasons, including divergence of the diffracted beams, if the source is not monochromatic, any adjustment problem of the diffractometers, as well as low absorption of the radiation by the sample. The instrumental broadening effect was determined to be $0.3^{\circ}$ using calibration standard Lanthanum Hexaboride $\left(\mathrm{LaB}_{6}\right)$. The value of $B$ in the above equation was calculated as:

$B=\left(b_{s}^{2}-b_{i}^{2}\right)^{1 / 2}$

where $b_{s}$ is the measured broadening of the chosen reflection and $b_{i}$ is the instrumental effect. ${ }^{23}$

\section{RESULTS AND DISCUSSION}

All the WAXS results, the dimensions of the crystallites and the crystallinity indices for the powder samples and the freeze-dried hydrogels classified by the different experimental and calculation modes are given in Table 1.

The X-ray diffraction patterns for both powder celluloses are illustrated in Figure 3. They reveal a structure of cellulose $\mathrm{I}^{28}$ The position of the major diffraction peak is $2 \theta \sim 22.96^{\circ}$ assigned to cellulose I reflection 200. Other main reflections are at $2 \theta \sim 15^{\circ}, \sim 16.5^{\circ}$ and $\sim 34^{\circ}$. These peaks are assigned to $1-10,110$ and 004 cellulose I reflections, respectively. The reflections in the spectrum of flax powder seem to be more clearly resolved and some reflections are shifted, compared to those of hardwood pulp powder. This revealed a more ordered supramolecular structure of the flax sample, compared to that of the hardwood pulp sample.

These considerations were confirmed by the calculation of the crystallinity values. The crystallinity of hardwood pulp powder was calculated by the amorphous fitting and amorphous subtraction methods to be $46 \%$ ( $\pm 3 \%$ and $\pm 2 \%$, respectively) and $74 \%( \pm 5 \%)$ by the peak height method. A similar variation can be seen for the crystallinity of the flax powder, so that the highest crystallinity value was determined by the peak height method to be $80 \%( \pm 5 \%)$. 
SHAHRIAR KARIM SAUROV et al.

Table 1

Crystallinity values computed using various methods and crystallite sizes for the powder samples and the freeze-dried hydrogels

\begin{tabular}{|c|c|c|c|c|c|}
\hline \multirow[b]{2}{*}{$\mathrm{N}$} & \multirow[b]{2}{*}{ Samples } & \multicolumn{3}{|c|}{ Crystallinity values, \% } & \multirow{2}{*}{$\begin{array}{c}\text { Crystal size } \\
\text { (width), } \\
( \pm 2 \AA)\end{array}$} \\
\hline & & $\begin{array}{l}\text { Amorphous } \\
\text { fitting } \\
\text { ( } \pm 3 \% \text { unit) }\end{array}$ & $\begin{array}{l}\text { Amorphous } \\
\text { subtraction } \\
\text { ( } \pm 2 \% \text { unit) }\end{array}$ & $\begin{array}{l}\text { Peak height } \\
\text { (Segal) method, } \\
( \pm 5 \% \text { unit })\end{array}$ & \\
\hline 1 & $\begin{array}{l}\text { Hardwood pulp } \\
\text { powder (HP) }\end{array}$ & 46 & 46 & 74 & 45 \\
\hline 2 & $\begin{array}{l}\text { HP regenerated } \\
\text { as hydrogel and } \\
\text { freeze-dried }\end{array}$ & 29 & 29 & - & - \\
\hline 3 & Flax powder $(\mathrm{FP})$ & 57 & 57 & 80 & 58 \\
\hline 4 & $\begin{array}{l}\text { FP regenerated as } \\
\text { hydrogel and } \\
\text { freeze-dried }\end{array}$ & 30 & 30 & - & $\begin{array}{c}28(1-10) 34 \\
(110)\end{array}$ \\
\hline
\end{tabular}

Crystallite sizes were computed based on cellulose I reflection 200 for samples 1 and 3 and cellulose II reflections 1 -10 and 110 for sample 4
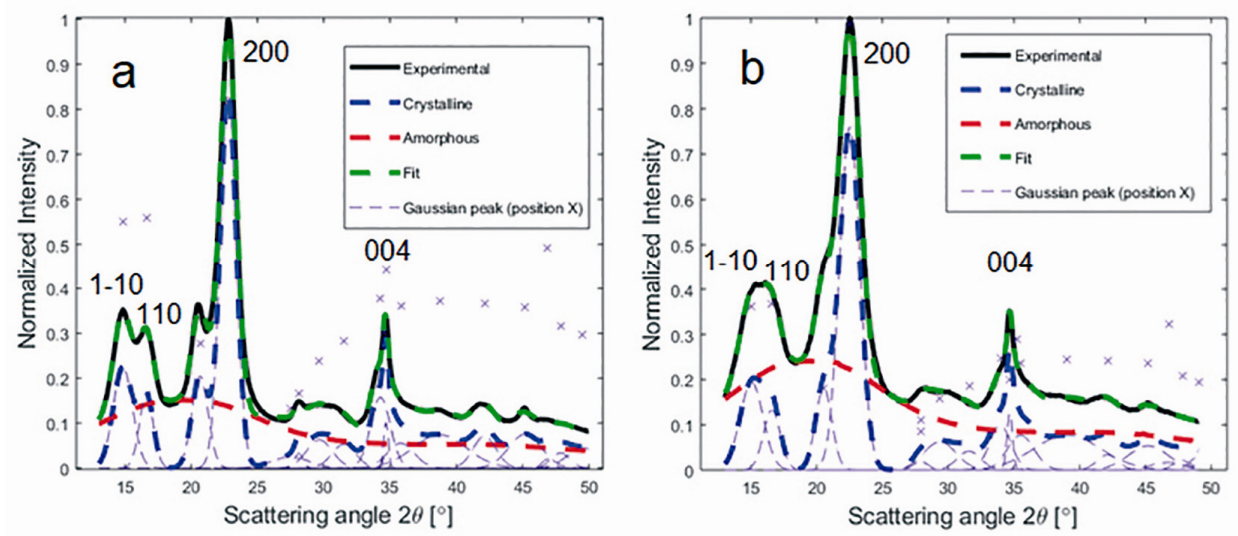

Figure 3: X-ray diffraction patterns and crystallinity computation using the amorphous fitting method for (a) flax powder cellulose and (b) hardwood pulp powder cellulose

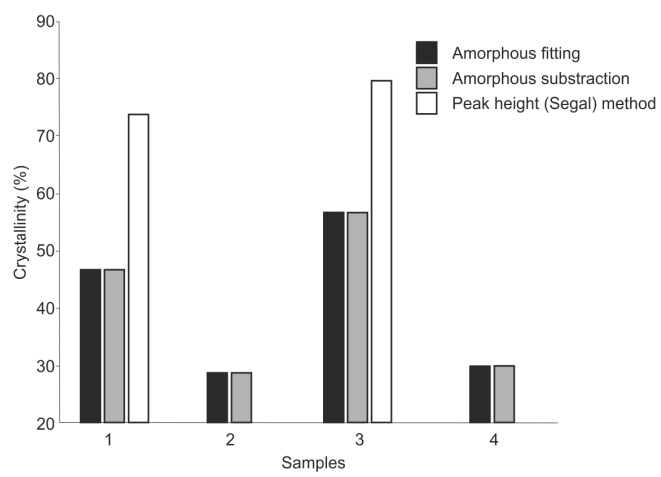

Figure 4: Variation of the crystallinity values for the samples listed in Table 1

The amorphous fitting and amorphous subtraction produced values of $57 \%( \pm 3 \%$ and $\pm 2 \%$, respectively). The crystallinity values for the flax powder sample were higher than those for hardwood pulp. The difference in values ranged from 8 to $\sim 20 \%$ (Table 1) depending on the computation method. The variation between the crystallinity results of the samples obtained by different methods is presented in Figure 4. 
The highest values were obtained using the peak height method compared to the values obtained by the amorphous fitting and subtraction methods. The difference was of $23 \%$ units for flax powder and $27 \%$ units for hardwood pulp powder. The amorphous fitting and amorphous subtraction methods gave similar values with negligible variation, as also predicted by other researchers. ${ }^{16}$

The crystallite width values slightly differed for the powder samples and were of $45 \pm 2 \AA$ and $58 \pm 2 \AA$ for the HP sample and FP sample, respectively. This also corresponded to the crystallinity values: larger crystallite width corresponded to higher crystallinity.

The diffraction patterns of the super-swollen hydrogel samples (Fig. 5) resembled the diffraction pattern of water. This is explained by the fact that cellulose hydrogels are threedimensional materials consisting of more than $97 \%$ water of the total mass, due to their high porosity and water absorbing ability to fill the empty spaces between the long chains. The hydrogel prepared from flax powder had a porosity value of 98.9 mass\% and maximum equilibrium water content of 2500 mass \%. For the hydrogel prepared from hardwood pulp powder, these parameters were 97.4 mass $\%$ and 2800 mass\%, respectively. ${ }^{25}$ Monitoring the long-term stability of the hydrogels showed that they maintained a stable 3D structure without collapsing when stored in water and ethyl alcohol at the temperature ranging from $20{ }^{\circ} \mathrm{C}$ to $70{ }^{\circ} \mathrm{C}$ during periods from 1 day to 180 days. We concluded on the basis of the results that the super-swollen hydrogels were stable in these media as long as needed because the swelling and absorbing capacity of the hydrogels did not change under these exposure conditions. Similar results have been obtained mostly for hydrogels prepared from cellulose nanoparticles, cellulose derivatives and/or composites with the aid of different cross-linkers. ${ }^{29-31}$

The mechanism of the above phenomenon was proposed in our earlier publication. ${ }^{7}$ The gelation and formation of the hydrogels were initiated by the solvent and water, and triggered with the rupture of the network of intra- and intermolecular H-bonds in the initial chains of the cellulose powders and the release of free chains in the solution of DMAc/LiCl. Additionally, the degree of polymerization of cellulose decreased and some $\mathrm{H}^{+}$atoms were substitued with $\mathrm{Cl}^{-}$ atoms, herewith the complex structure $\mathrm{Li}^{+}(\mathrm{DMAc})_{\mathrm{x}}$ was formed. In this solution, the cellulose chains were strongly solubilized with molecules of the solvent and a gel containing a high volume of the solvent was generated. The formation of the gel revealed that the solubilized cellulose chains became available to reconstruct a new network of H-bonds accompanied by an increase in the concentration of cellulose in the gel. After rinsing and replacing the solvent with water, the hydrogel was obtained. The shape and stability of the hydrogels corresponded to the strong interaction of water molecules with $\mathrm{OH}$ groups and to the formation of the novel network of $\mathrm{H}$-bonds between the $\mathrm{OH}$ groups and water. In this case, the water molecules bound to the $\mathrm{OH}$ groups of the cellulose were randomly arranged in the volume of the hydrogel (Fig. 6, a). Both processes, gelation and formation of the hydrogel, were performed by spontaneous self-assembly and aggregation of the cellulose chains under ambient conditions, without any changing temperature and air pressure and without any antisolvent. As it was mentioned, the prepared hydrogels were stable in water as long as needed.

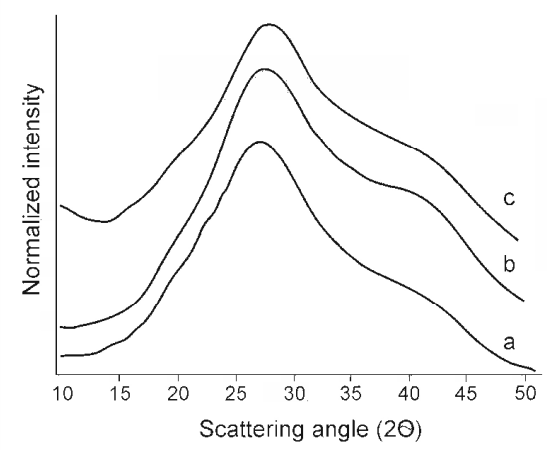

Figure 5: X-ray diffraction patterns for (a) super-swollen hydrogel sample from Flax powder, (b) super-swollen hydrogel sample from Hardwood pulp powder and (c) water 


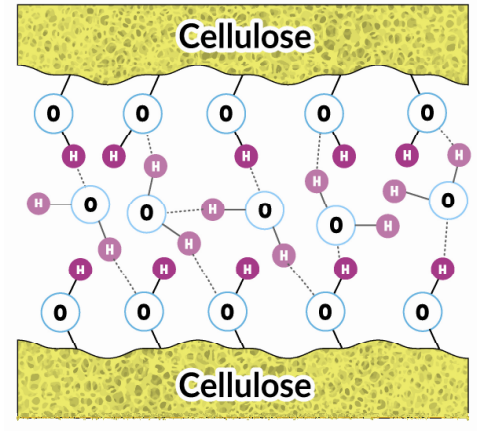

a

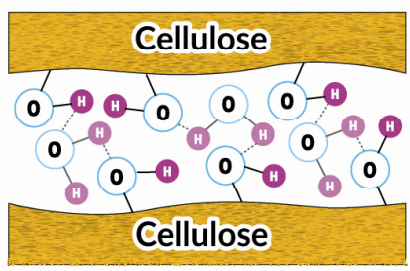

b

Figure 6: Schematic images of (a) H-bonding between cellulose and water molecules in the newly formed superswollen hydrogel that contained a large amount of water and (b) in the partially dried hydrogel

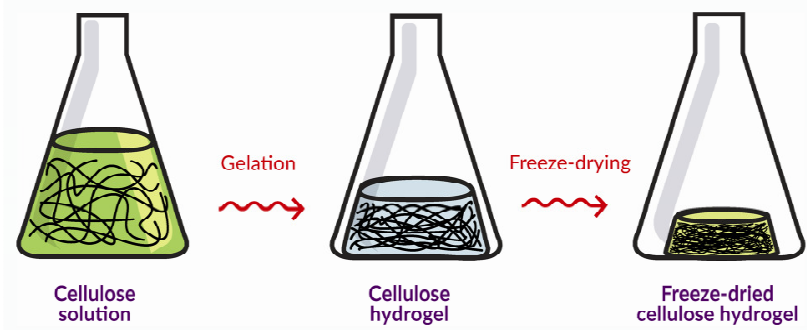

Figure 7: Schematic images of the cellulose solution, cellulose hydrogel and freeze-dried cellulose hydrogel

However, the stable hydrogels collapsed while freeze-drying and formed rigid slightly accessible structures (Fig. 2, c). The water molecules bound to the $\mathrm{OH}$ groups of cellulose are still arranged in the volume (Fig. 6, b), but are more tightly packed in the freeze-dried hydrogel. This is also illustrated schematically in Figure 7.

In discussing these issues, we took into account that the interaction of water with cellulose was investigated in many studies and we refer to some of them herein. ${ }^{32-34}$ Thus, as described in a previous study, ${ }^{33}$ the organization of water molecules adsorbed on the cellulose surface or in the bulk and the supramolecular hydrated structure of cellulose chains and/or microfibril aggregates represents one of the complex problems in the physical chemistry of natural polymers. One complication in understanding the mechanisms of moisture sorption or water interaction with cellulose and its dependence on the structure is the complexity of the cellulose structure. In most studies, hydration and dehydration were studied and as a result of experiments, they induced structural and morphological changes of native cellulose fibres. ${ }^{4}$ In the case of this study, the consideration of the hydrogel structure and interaction of the hydrogels with water, with the aid of the widely recognized mechanism of the hydration, could not be completely used as in the case of cellulose fibres, nanocellulose or cellulose derivatives subjected to the targeted action with water. We studied materials that were independently generated from the solutions and had a definite structure reformed under the regeneration. Our purpose was to define this structure with the reliable X-ray method.

The diffraction patterns of the freeze-dried hydrogels are presented in Figures 8 and 9. The peaks located at $2 \theta \sim 12^{\circ}, \sim 20^{\circ}, \sim 21^{\circ}$ and $\sim 34^{\circ}$ appeared. These peaks were attributed to the cellulose II polymorph. The appearence of the peak at $2 \theta \sim 34^{\circ}$ for the cellulose II polymorph has been also observed after mercerisation of the cellulose, due to the treatment with solutions of $\mathrm{NaOH}$ with different concentrations. ${ }^{35}$ These peaks have also been found for many cellulose samples subjected to dissolution in various solvents and subsequent regeneration from the solutions, which were transformed to gels and to multiple shaped materials. ${ }^{36,37}$ There can be observed interesting differences between the scattering patterns of the freeze-dried hydrogels from flax and hardwood pulp: the peaks attributed to cellulose II can be seen more clearly in the freeze-dried hydrogel from flax (Fig. 9). Based on these patterns, the conversion of cellulose I to cellulose II may have occurred to a greater extent 
in the freeze-dried hydrogel from flax, compared to hardwood pulp.

The crystallinity values of the freeze-dried hydrogels are also listed in Table 1. The crystallinity of the freeze-dried hydrogel from hardwood pulp powder calculated both by the amorphous fitting and amorphous subtraction methods was $29( \pm 3) \%$ The crystallinity of the freeze-dried hydrogel from flax powder calculated by the same methods was $30( \pm 3) \%$. So, in this case, the crystallinity values calculated by the amorphous fitting and amorphous subtraction methods were almost the same. Attention should be paid to the fact that the crystallinity values of both freeze-dried hydrogel samples were much lower than the crystallinity of the powder samples.

While calculating the crystallinity values of the freeze-dried hydrogels (both hydrogels from hardwood pulp powder and flax powder), the peak height method was not used. As described earlier, this method considers the reflection 020 in the case of cellulose II. In this study, the 110 and 020 peaks overlap significantly, making it difficult to separate individual peaks (see Fig. 9). Also, in this case, the minimum intensity needed for the peak height method is impossible to be determined unambigiously.
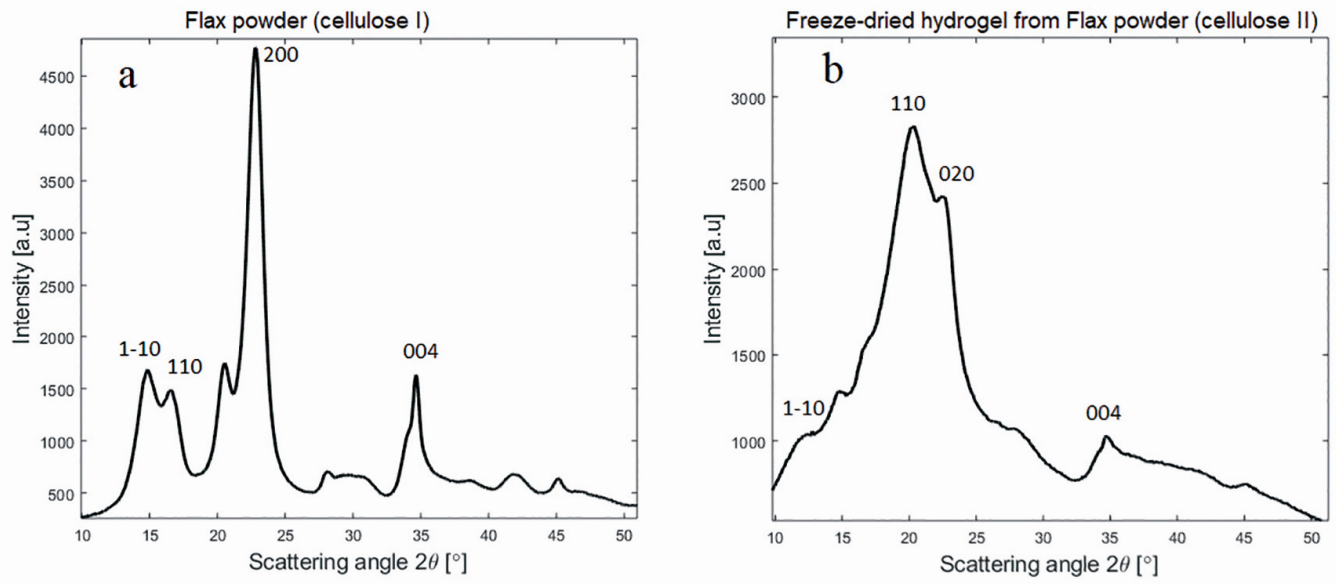

Figure 8: Comparison of WAXS patterns between flax powder (a) and freeze-dried hydrogel from flax powder (b)
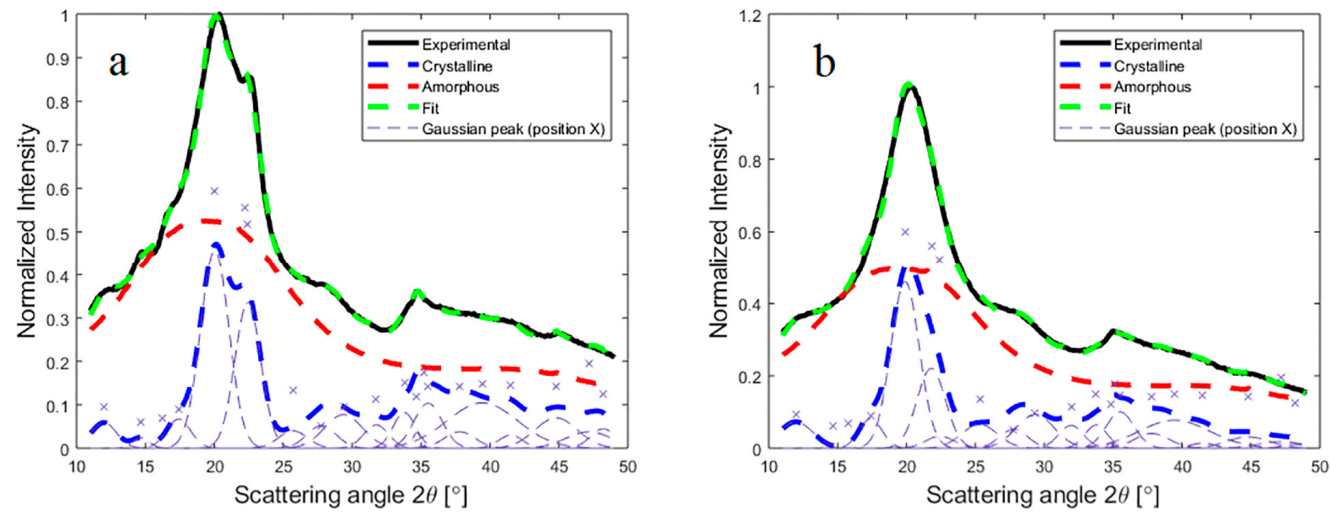

Figure 9: WAXS patterns for freeze-dried hydrogels (a) from flax powder and (b) from hardwood pulp powder

Also, due to the heavy overlapping of the cellulose II peaks, the width of the crystallites of the freeze-dried hydrogel from hardwood pulp could not be determined reliably (see Fig. 9b, where only one wide (Gaussian) peak without any details of underlying two main cellulose II reflections (described by two Gaussians) is observed). Whereas for the freeze-dried hydrogel from flax, the cellulose II reflections $1-10$ and 110 could be analyzed; based on that, the crystallite width in the freeze-dried flax hydrogel was $28 \pm 2$ $\AA$, along the $1-10$ direction and $34 \pm 2 \AA$ along the 
110 direction. This crystallite size is significantly smaller, compared to the pristine flax powder (cellulose I) sample.

A number of authors showed that when powder samples with the structure of cellulose I were dissolved in $\mathrm{DMAc} / \mathrm{LiCl}$ and regenerated from the solutions as hydrogels, irrespective of the type of the solvent and regeneration mode, they turned to more thermodynamically stable polymorphs. ${ }^{4,38,39}$ Also, in this study, the diffraction patterns revealed changes in the peak intensities at different cellulose reflections and polymorphic transformation of the allomorphs from cellulose I to cellulose II. The transformation of the crystal structure from cellulose I to the cellulose II polymorph provided large changes in the parameters of the cellulose crystallites. The parameters of the crystallites, namely, the crystal width and the crystallinity values changed. Also, interesting differences could be observed between the WAXS patterns of the dried hydrogel samples made of different plant materials (flax vs hardwood pulp), i.e. the crystalline structure was not the same in these two regenerated samples. The structural order decreased dramatically. The structure of cellulose changed subsequently: high order (powder cellulose I) $\rightarrow$ disordered chains (solution) $\rightarrow$ partial order (regenerated cellulose II).

It follows from these results that the arrangement, the environment and, accordingly, the system of hydrogen bonds between the cellulose chains in cellulose II crystallites has changed so dramatically that the crystalline order of cellulose I was severely disturbed. Taking into account the obtained results, it is difficult to imagine that the cellulose chains can be restored to the pristine crystalline state of cellulose I or to another highly crystalline state. There are some publications where the authors described the formation of regenerated cellulose I from the solutions. However, it is mostly approved that cellulose I chains, which were parallel-arranged, changed their direction and turned to an antiparallel order in cellulose II. ${ }^{40-42}$ The anti-parallel arrangement is thermodynamically more favourable than the parallel one. ${ }^{43}$ The hydrogen bonding in cellulose II is more complicated than in cellulose I and the anti-parallel chain model enables the formation of not only inter-chains, but also of inter-plane hydrogen bonds. ${ }^{43}$ In our opinion, this may be one of the reasons providing greater stability to the structure of cellulose II regenerated from the solutions. However, we could not determine the crystallinity values and the crystal size of the cellulose in the superswollen hydrogels, because of the low concentration of cellulose.

Based on our results, additional investigations are necessary to understand the rearrangement of the cellulose chains of the pristine cellulose I dissolved in solutions to some sort of organized structure of cellulose II during the regeneration from the solutions in the form of hydrogels made from different plant origins.

\section{CONCLUSION}

The calculation mode had a strong impact on the obtained crystallinity values of cellulose. The highest values were obtained using the peak height method, whereas the amorphous subtraction and the amorphous fitting generated similar values. The crystallinity values of the cellulose powder from hardwood pulp varied from $46 \%$ to $74 \%$ and those of the cellulose powder from flax fibers varied from $57 \%$ to $80 \%$, depending of the calculation mode. These powder samples had crystal widths in the range of 45 and $58 \AA$. However, the freeze-dried hydrogels exhibited a partly crystalline structure and smaller crystallite width. The significant difference in the crystallinity values of the powder celluloses and the freeze-dried hydrogels was due to the transformation of cellulose I to cellulose II polymorphs. This transformation impacted considerably the parameters of the crystallites. Generally, the structure of cellulose changed consequently: high order (powder cellulose I) $\rightarrow$ disordered chains (solution) $\rightarrow$ partial order (regenerated cellulose II).

The application of the three various calculation modes showed that a definite standardization is necessary to characterize the parameters of cellulose crystals.

Conflicts of interests: The authors declare that they have no conflict of interests.

\section{REFERENCES}

1 "Cellulose: Molecular and Structural Biology: Selected Articles on the Synthesis, Structure and Application of Cellulose", edited by R. M. Brown and I. M. Saxena, Springer Science \& Business Media, 2007, https://doi.org/10.1007/978-1-4020-5380-1

2 C. Chang and L. Zhang, Carbohyd. Polym., 84, 40 (2011), https://doi.org/10.1016/j.carbpol.2010.12.023

X. Qiu and S. Hu, Materials, 6, 738 (2013), https://doi.org/10.3390/ma6030738 
4 B. Medronho and B. Lindman, Curr. Opin. Colloid Interface $\quad$ Sci., $\quad \mathbf{1 9}, \quad 32 \quad$ (2014), https://doi.org/10.1016/j.cocis.2013.12.001

5 S. Raymond, A. Kvick and H. Chanzy, Macromolecules, 28, $8422 \quad$ (1995), https://doi.org/10.1021/ma00128a063

6 C. Zhang, R. Liu, J. Xiang, H. Kang, Z. Liu et al., J. Phys. Chem. B, 118, 9507 (2014), https://doi.org/10.1021/jp506013c

7 N. Kotelnikova, A. Mikhailidi, Yu. Martakova and S. Andersson, Cellulose Chem. Technol., 50, 545 (2016), https://doi.org/10.32964/tj17.02.81

8 Y. Sekiguchi, C. Sawataria and T. Kondo, Carbohyd. Polym., 53, $145 \quad$ (2003), https://doi.org/10.1016/s0144-8617(03)00050-x

9 S. Beck-Candanedo, M. Roman and D. G. Gray, Biomacromolecules, $\quad$ 6, $1048 \quad$ (2005), https://doi.org/10.1021/bm049300p

10 Y. Qing, Y. Wu, Z. Cai and X. Li, J. Nanomater., ID 594734 (2013), https://doi.org/10.1155/2013/594734

11 J. Watanabe, Y. Kiritoshi, K. W. Nam and K. Ishihara in "Encyclopedia of Biomaterials and Biomedical Engineering", edited by G. E. Wnek and G. L. Bowlin, Marcel Dekker Inc., New York, 2004, pp. 1439-1451, https://doi.org/10.1201/b18990-139

12 H. Saito, A. Sakurai, M. Sakakibara and H. Saga, Appl. Polym. Sci., 90, $3020 \quad$ (2003), https://doi.org/10.1002/app.13015

13 D. Ishii, D. Tatsumi, T. Matsumoto, K. Murata, H. Hayashi et al., Macromol. Biosci., 6, 293 (2006), https://doi.org/10.1002/mabi.200500231

14 P. Singh, H. Duarte, L. Alves, F. Antunes, N. Le Moigne et al., in "Cellulose - Fundamental Aspects and Current Trends", edited by M. Poletto, University of Caxias Do Sul, Brazil, 2015, https://doi.org/10.5772/61402

15 P. Ahvenainen, I. Kontro and K. Svedström, Cellulose, 23, 1073 (2016), https://doi.org/10.1007/s10570-016-0881-6

16 N. Terinte, R. Ibbett and K. Schuster, Lenzinger Berichte, $\quad \mathbf{8 9}, \quad 118 \quad$ (2011), https://www.lenzing.com/index.php?type=88245\&tx_fi ledownloads_file $\% 5$ bfileName $\% 5 \mathrm{~d}=$ fileadmin/content/ PDF/03_Forschung_u_Entwicklung/Ausgabe_89_2011 .pdf

S. Park, J. O. Baker, M. E. Himmel, P. A. Parilla and D. K. Johnson, Biotechnol. Biofuel., 3, 10 (2010), https://doi.org/10.1186/1754-6834-3-10

18 L. Segal, J. J. Creely, A. E. Martin Jr. and C. M. Conrad, Textile Res. J., 29, 786 (1959), https://doi.org/10.1177/004051755902901003

19 C. P. Azubuike, H. Rodriguez, A. O. Okhamafe and R. D. Rogers, Cellulose, 19, 425 (2012), https://doi.org/10.1007/s10570-011-9631-y

20 S. Nam, A. D. French, B. D. Condon and M. Concha, Carbohyd. Polym., 135, 1 (2016), https://doi.org/10.1016/j.carbpol.2015.08.035
21 W. Ruland, Acta Crystallogr., 14, 1180 (1961), https://doi.org/10.1107/s0365110x61003429

22 S. Maunu, T. Liitiä, S. Kauliomäki, B. Hortling and J. Sundquist, Cellulose, 7, $147 \quad$ (2000), https://doi.org/10.1023/a:1009200609482

23 S. Andersson, R. Serimaa, T. Paakkari, P. Saranpää and E. Pesonen, J. Wood Sci., 49, 531 (2003), https://doi.org/10.1007/s10086-003-0518-X

${ }^{24}$ K. Leppänen, S. Andersson, M. Torkkeli, M. Knaapila, N. Kotelnikova et al., Cellulose, 16, 999 (2009), https://doi.org/10.1007/s10570-009-9298-9

25 A. Mikhailidi, S. Karim Saurov, S. Andersson and N. Kotelnikova, Tappi J., 17, 81 (2018), https://doi.org/10.32964/tj17.02.81

26 P. Langan, Y. Nishiyama and H. Chanzy, Biomacromolecules, $\quad \mathbf{2 ,} 410 \quad$ (2001), https://doi.org/10.1021/bm005612q

27 P. Scherrer in "Nachrichten von der Gesellschaft der Wissenschaften zu Göttingen", MathematischPhysikalische Klasse, Weidmannsche Buchhandlung, Berlin, 1918, pp. 98-100, http://eudml.org/doc/59018

28 Y. Nishiyama, P. Langan and H. Chanzy, J. Am. Chem. Soc., 124, $9074 \quad$ (2002), https://doi.org/10.1021/ja0257319

${ }^{29}$ S. M. Fijul Kabir, P. P. Sikdar, B. Haque, M. A. Rahman Bhuiyan, A. Ali et al., Progress Biomater., 7, 153 (2018), https://doi.org/10.1007/s40204-018-00950

30 M. R. Hossen, N. Dadoo, D. G. Holomakoff, A. Co, W. M. Gramlich et al., Polymer, 151, 231 (2018), https://doi.org/10.1016/j.polymer.2018.07.016

31 S. S. Nair, J. Y. Zhu, Y. Deng and A. J. Ragauskas, ACS Sustain. Chem. Eng., 2, 772 (2014), https://doi.org/10.1021/sc400445t

32 L. Fang and J. M. Catchmark, Cellulose, 21, 3951 (2014), https://doi.org/10.1007/s10570-014-0435-8

33 E. L. Lindh, C. Terenzi, L. Salmen and I. Furo, Phys. Chem. Chem. Phys., 19, 4360 (2017), https://doi.org/10.1039/c6cp08219j

34 A. Ch. Khazraji and S. Robert, J. Nanomater., 2013, 1 (2013), https://doi.org/10.1155/2013/745979

35 P. Mansikkamäki, M. Lahtinen and K. Rissanen, Cellulose, 12, $233 \quad$ (2005), https://doi.org/10.1007/s10570-004-3132-1

36 J. Obradovic, P. Fardim, L. Lassila, P. Navard and D. Kronlund, BioResources, 10, 2143 (2015), https://doi.org/10.15376/biores.10.2.2143-2155

37 J. Obradovic, H. Wondraczek, P. Fardim, L. Lassila and P. Navard, Cellulose, 21, 4029 (2014), https://doi.org/10.1007/s10570-014-0403-3

38 A. Isogai, in "Cellulosic Polymers - Blends and Composites", edited by R. D. Gilbert, Hanser Publishers, 1994, pp. 1-24, https://doi.org/10.1002/pi.1995.210370116

39 S. Pérez and K. Mazeau, in "Polysaccharides: Structural Diversity and Functional Versatility", edited by S. Dumitriu, Marcel Dekker Inc., 2005, pp. 41-68, https://www.crcpress.com/Polysaccharides-Structural- 
SHAHRIAR KARIM SAUROV et al.

Diversity-and-Functional-Versatility-

Second/Dumitriu/p/book/9780824754808

40 F. J. Kolpak and J. Blackwell, Macromolecules, 8, 563 (1975), https://doi.org/10.1021/ma60046a037

41 T. Okano and A. Sarko, J. Appl. Polym. Sci., 30, 325

https://doi.org/10.1002/app.1985.070300128 (1985)
42 F. J. Kolpak and J. Blackwell, Polymer, 19, 132 (1978), https://doi.org/10.1016/0032-3861(78)90028-9 43 Y. Wan, F. An, P. Zhou, Y. Li, Y. Liu et al., Chem. Commun., $\quad 53, \quad 3595 \quad$ (2017), https://doi.org/10.1039/c7cc00450h 